\title{
Multimodel Robust Control for Hydraulic Turbine
}

\author{
Jakub Osuský and Stanislav Števo \\ Institute of Control and Industrial Informatics, Faculty of Electrical Engineering and Information Technology, \\ Slovak University of Technology, Ilkovičova 3, 81219 Bratislava, Slovakia
}

Correspondence should be addressed to Jakub Osuský; jakub.osusky@stuba.sk

Received 4 April 2014; Accepted 1 August 2014; Published 25 August 2014

Academic Editor: Mamun B. Ibne Reaz

Copyright (C) 2014 J. Osuský and S. Števo. This is an open access article distributed under the Creative Commons Attribution License, which permits unrestricted use, distribution, and reproduction in any medium, provided the original work is properly cited.

\begin{abstract}
The paper deals with the multimodel and robust control system design and their combination based on $M-\Delta$ structure. Controller design will be done in the frequency domain with nominal performance specified by phase margin. Hydraulic turbine model is analyzed as system with unstructured uncertainty, and robust stability condition is included in controller design. Multimodel and robust control approaches are presented in detail on hydraulic turbine model. Control design approaches are compared and used for derivation of new approaches which combine advantages of both.
\end{abstract}

\section{Introduction}

When hydraulic turbine power control is considered, the water inertia effect is a factor that makes it difficult to maintain stability under isolated operation or to have fast response in case of load change in the whole operational range. Nonlinear models of hydraulic turbine control systems are needed in those cases where the turbine power varies in the whole range. The turbine model parameters vary significantly with the unpredictable load variations. Such nonlinearities make the controller design a nontrivial task due to the fact that a controller designed for specific operating conditions may not perform well under different loads [1]. This fact leads to the adaptive [2] or hybrid control algorithms.

There are several approaches to model hybrid systems [3]. In a model of a large class of hybrid systems, [4] is considered as discrete event system and continuous dynamic modeled by differential or difference equation. Such models are used to formulate a general stability analysis and controller synthesis framework for hybrid systems. Results for modeling and stability analysis of hybrid systems have been presented in $[3,5,6]$. In this paper, we follow the class of hybrid system known as switched systems [7].

In this paper, hybrid control, often denoted by multimodel control, will be used. Several switching algorithms will be compared to show how the switching speed affects performance and stability. Well-known robust control method will be presented as a control solution which ensures stability on whole operating range, but with lower performance. Combination of multimodel control and robust control which ensure stability and good performance will be presented on numerical example of nonlinear hydraulic turbine model.

\section{Preliminaries and Problem Formulation}

Consider nonlinear system as uncertain system described by set of transfer functions $G_{q}(s)$ and controller $R_{q}(s)$ in standard configuration:

$$
G_{q}(s) \in \Pi, \quad q=1,2, \ldots, N,
$$

where $y(s), u(s)$, and $w(s)$ are output, control, and setpoint variables of system, respectively; $q=1,2, \ldots, N$ is the number of linear transfer functions describing uncertain system and also the number of controllers. Hence, for each identified transfer function, one controller is designed.

To deal with uncertainties instead of a single model, the behavior of a class of models $\Pi$ is considered.

Multimodel control is based on several linear models identified in operating points. For each linear model, local 


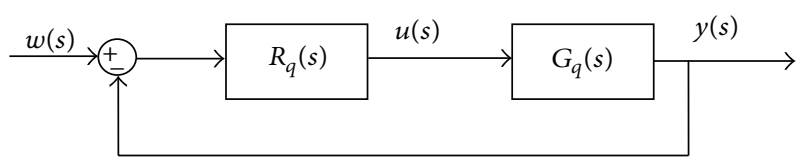

FIGURE 1: Standard feedback control configuration.

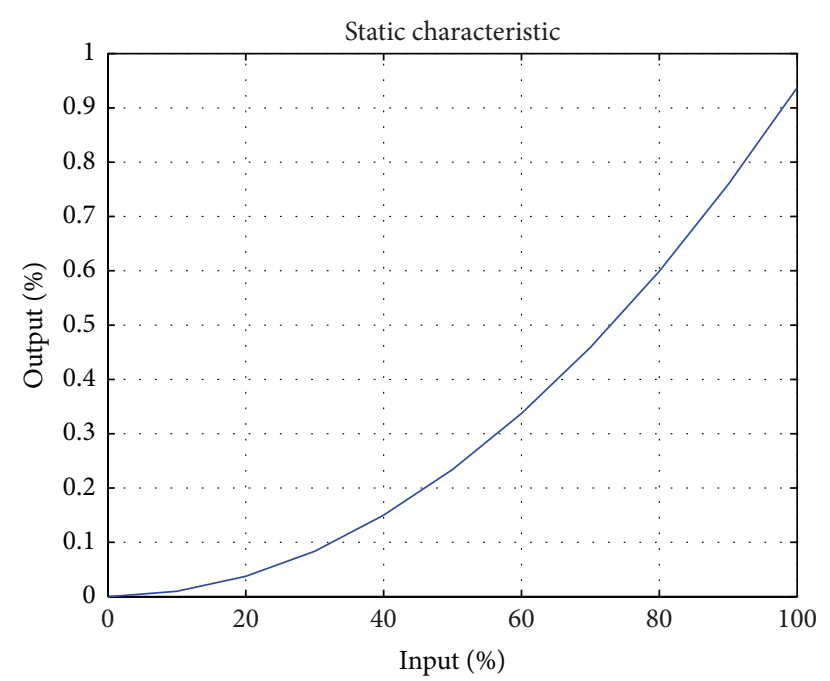

FIGURE 2: Static characteristic of nonlinear system.

controller is designed which ensures stability and perhaps even performance only for this model. As nonlinear system moves from one operating point to another, controller switches from one local controller to another one. Performance of this control approach can be much better as using only one controller on whole range, but this method does not yield stability when nonlinear system is between operating points. Fast switching between local controllers can decrease performance and also lead to instability.

In robust control, several linear models identified in operating points are used for uncertainty model calculation. Then, for system control, one controller is used, which ensures stability on whole operating range, between all operating points. Performance (of robust control) is not so good as performance of multimodel control; however, there is no problem with stability by controllers switching. When nonlinearity is increased, uncertainty is increased too and performance of robust control decreases. If the system is strongly nonlinear, uncertainty could be too big to be able to control the system using robust control.

In this paper, designing a set of local controllers similar to those in multimodel control design will be done, but each local controller will be designed as robust to ensure stability between operating points. By robust design of local controller, instead of all operating points, only given and neighbourhood operating points will be considered. This type of control will be called robust multimodel control and will ensure stability and higher performance than robust control.

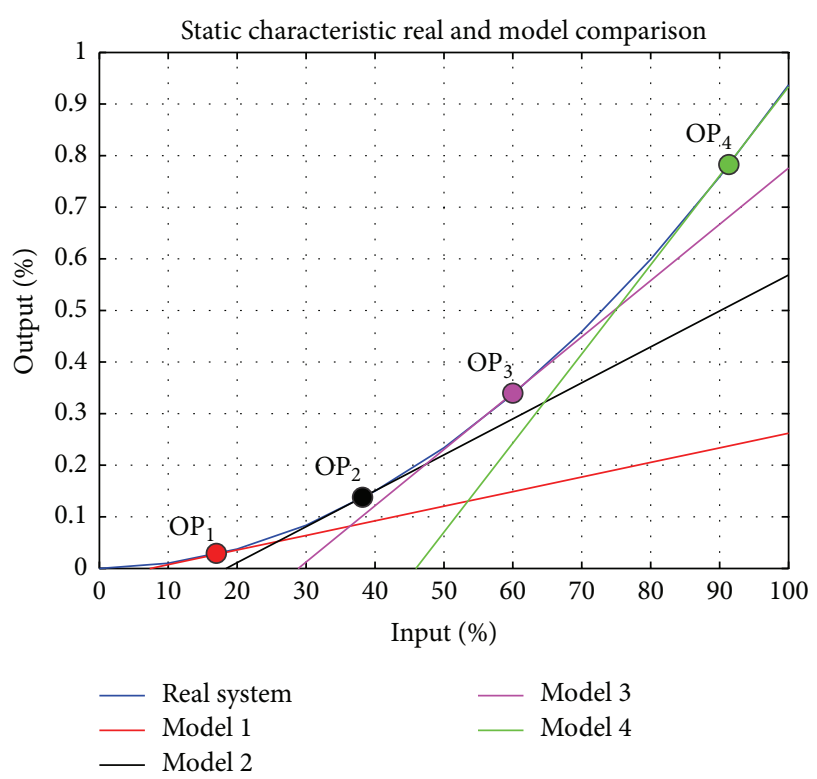

FIGURE 3: Static characteristic with linear models.

\section{Theoretical Results}

3.1. Multimodel Control Design. Consider a system with nonlinear static characteristic (Figure 2).

Static characteristic is nonlinear and could be approximate using $m$ linear models (in our case four models, $m=4$, Figure 3).

These models are simple to obtain using input-output identification. For each model (operating point (OP)), local PID controller can be tuned using any well-known and tested method. Depending on system output, multimodel controller switches between local PID controllers. Local controllers ensure stability only in operating point.

Characteristic equation of closed-loop feedback system (Figure 1) can be written as follows:

$$
1+R_{q}(s) G_{q}(s)=0 .
$$

Equation (2) can be rewritten into polynomial form:

$$
p_{q}(s)=p_{0}+p_{1} s+p_{2} s^{2}+\cdots+p_{n} s^{n}=0,
$$

where $q$ is index of operating point.

Local controllers ensure only stability (negative roots) of (3) for $q=1,2, \ldots, m$. For stability of the whole system, segments

$$
\begin{gathered}
p s_{i}(s, \chi)=(1-\chi) p_{i}(s)+\chi p_{i+1}(s), \\
\chi \in\langle 0,1\rangle, \quad i=1,2, m-1
\end{gathered}
$$

between operation points must be stable too, independent of switching speed. One way how to reach segment stability is using of robust control.

3.2. Robust Control Design. A simple uncertainty model is obtained using unstructured model approach. In the sequel 


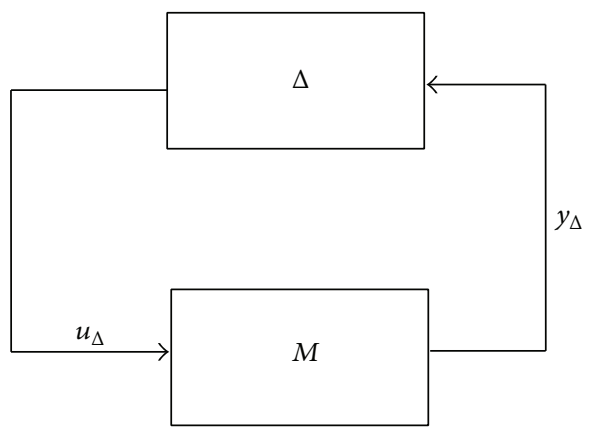

FIgURe 4: $M-\Delta$ structure.

(see Figure 5), two most common single uncertainty models are considered, additive and input multiplicative model type [8]. Corresponding classes of uncertain models are given as follows.

(1) Additive uncertainty model for operating points $q=$ $1,2, \ldots, m$ :

$$
G_{q}(s)=G_{0}(s)+\Delta_{a}(s) W_{a}(s),
$$

where

$$
\begin{gathered}
l_{a}(\omega)=\max _{G_{q} \in \Pi} \sigma_{M}\left[G_{q}(s)-G_{0}(s)\right], \quad q=1,2, \ldots, m, \\
\left|W_{a}(s)\right| \geq l_{a}(s) .
\end{gathered}
$$

(2) Input multiplicative uncertainty model for operating points $q=1,2, \ldots, m$ :

$$
G_{q}(s)=G_{0}(s)\left(I+\Delta_{i}(s) W_{i}(s)\right),
$$

where

$$
\begin{aligned}
& l_{i}(\omega)=\max _{G_{q} \in \Pi} \sigma_{M}\left[G_{0}(s)^{-1}\left(G_{0}(s)-G_{q}(s)\right)\right], \\
& q=1,2, \ldots, m, \\
&\left|W_{i}(s)\right| \geq l_{i}(s), \quad \sigma_{M}\left(\Delta_{a}(s)\right) \leq 1, \quad \sigma_{M}\left(\Delta_{i}(s)\right) \leq 1, \\
& \forall s \in D,
\end{aligned}
$$

where $D$ is Nyquist contour [8].

Standard feedback configuration with unstructured uncertainty of any type can be rearranged to obtain the general $M-\Delta$ structure in Figure 4 where $M(s)$ represents the transfer function matrix (containing controller $R(s)$ nominal model $\left.G_{0}(s)\right)$ and $\Delta(s) ; \sigma_{\max }[\Delta(j \omega)] \leq 1$ is the normalized perturbation.

Robust stability condition for unstructured perturbations is formulated in terms of stability of the $M-\Delta$ system: if both the nominal system $M(s)$ (nominal stability) and the normalized perturbation $\Delta(s)$ are stable, closed-loop stability is guaranteed for

$$
\sigma_{\max }[M(j \omega)]<1, \forall \omega
$$

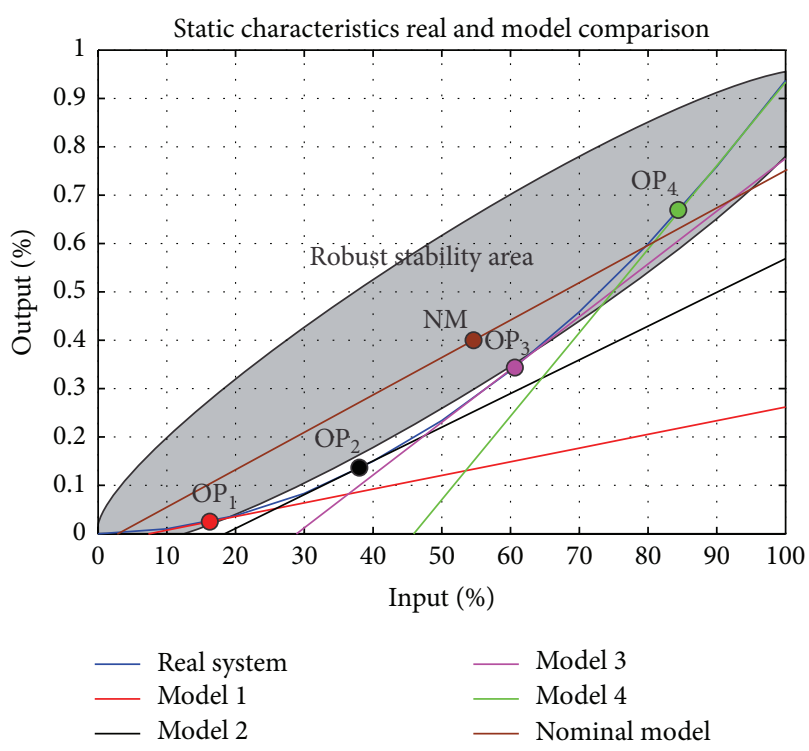

FIGURE 5: Illustration of how the full operating area of nonlinear system is covered by stability area of single robust controller.

(i) for additive uncertainty:

$$
M_{a}(s)=-\left[I+R(s) G_{0}(s)\right]^{-1} R(s),
$$

(ii) for input multiplicative uncertainty:

$$
M_{i}(s)=-\left[I+R(s) G_{0}(s)\right]^{-1} R(s) G_{0}(s) .
$$

In view of (11) and (12), condition (10) reads as follows:

$$
\sigma_{\max }\left[M_{k}(s)\right]<\frac{1}{\left|l_{k}(s)\right|}, \quad k=a, i .
$$

Robust control approach based on unstructured uncertainty and with robust stability ensured using $M-\Delta$ structure can be summarized as follows.

(a) Nominal model calculation: nominal model $G_{0}(s)$ is calculated as average transfer function from all $m$ operating points $G_{q}(s), q=1,2, \ldots, m$.

(b) For nominal model standard feedback controller is designed: good choice could be controller design method with desired phase margin.

(c) Robust stability verification according to (13): if robust stability is not passed, part (b) must be done again. Increasing phase margin by controller design is possible to improve robust properties of controller.

Note: segments (4) are inclusive of robust stability area.

3.3. Robust Multimodel Control Design. In this case, for each operating point of switched system, with arbitrary switch algorithm, robust controller $R_{q}(s), q=1,2, \ldots, m$ is designed. 
Controller guarantees the robust stability and performance for all operating points and over the entire operating range specified by uncertain models (5) or (7).

Let derive robust stability condition for operating point q. The nominal model $G_{0}$ and uncertainty model will be calculated from transfer functions $G_{q n}(s), q n=q-1, q, q+1$; $q=1,2, \ldots, m$. Because of switching the process from mode $q$, the plant may be switched to neighbourhood operating point $q+1=1,2, \ldots, m$ or $q-1=1,2, \ldots, m$; to guarantee the robust stability for such switching algorithm, (5) and (7) need to be modified as follows:

$$
G_{q n}(s)=G_{0 n}(s)+W_{q n \_a}(s) \Delta_{q n \_a}(s),
$$

where

$$
\begin{gathered}
l_{q n a}(\omega)=\max _{G_{q n}(s) \in \Pi} \sigma_{M}\left(G_{0 n}(s)-G_{q n}(s)\right), \\
q n=q-1, q, q+1 ; \quad q=1,2, \ldots, m, \\
\left|W_{q n a}(s)\right| \geq l_{q n a}(\omega),
\end{gathered}
$$

and for input multiplicative case,

$$
G_{q n}(s)=G_{0 n}\left(I+\Delta_{q n \_i}(s) W_{q n \_i}(s)\right),
$$

where

$$
\begin{gathered}
l_{q n i}(\omega)=\max _{G_{q n}(s) \in \Pi} \sigma_{M} G_{q n}(s)^{-1}\left(G_{0 n}-G_{q n}(s)\right) \\
q n=q-1, q, q+1 ; \quad q=1,2, \ldots, m .
\end{gathered}
$$

Robust controller design procedure for each operating point $q=1,2, \ldots, m$ of switched system goes the same way as for robust control design but instead of all operating points only given and neighbourhood operating points are considered.

Robust multimodel control approach based on unstructured uncertainty and with robust stability ensured using $M-\Delta$ structure can be summarized as follows.

(a) For each operation point, nominal model is calculated using not all but only neighborhood operating points. Nominal model $G_{0 n}(s)$ is for $q$ th operating point calculated as average one from $G_{q}(s), G_{q-1}(s), G_{q+1}(s)$ (if $q=1$ only $G_{q}(s)$ and $G_{q+1}(s)$ are considered. If $q=m$ only $G_{q}(s)$ and $G_{q-1}(s)$ are considered).

(b) For nominal model $G_{0 n}(s)$ standard feedback controller is designed. Good choice could be controller design method with desired phase margin.

(c) Robust stability verification according to (13): if robust stability is not passed, part (b) must be done again. Increasing phase margin by controller design is possible to improve robust properties of controller.

These three steps have to be done $m$ times once for each operating point.

Stability areas of local controllers (Figure 6) are overlapping so nonlinear system is stable also between operating points. Robust stability area is smaller like using only one robust controller which will yield less conservative solutions.

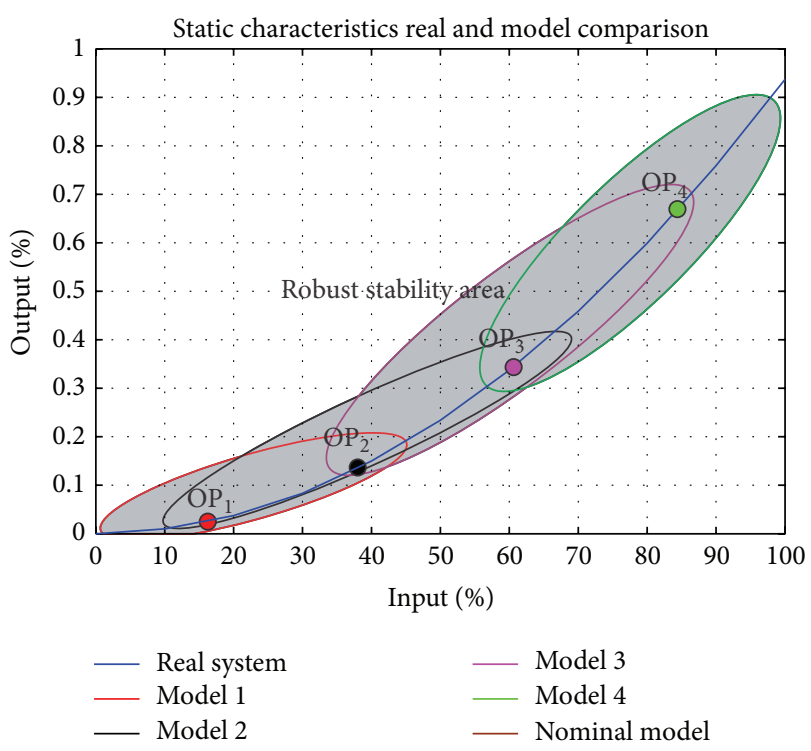

FIGURE 6: Illustration of how the full operating area of nonlinear system is covered by stability area of multimodel robust controller.

\section{Case Study}

Consider hydraulic turbine with power operating range (0$100) \%$. In our case, nonlinear model described in [1] will be identified using input-output identification in five operating points (output power of $20 \%, 40 \%, 60 \%, 80 \%$, and $90 \%$ ). As identification results linear models in transfer functions form will be obtained.

Verification of linear models identified in operating points can be found in appendix (Figures 16, 17, 18, 19, and 20). Transfer functions of these models are as follows:

$$
\begin{gathered}
G_{1}(s)=\frac{-1.516 s+2.46}{s^{2}+2.597 s+2.474}, \\
G_{2}(s)=\frac{-1.911 s+1.536}{s^{2}+1.994 s+1.554}, \\
G_{3}(s)=\frac{-1.931 s+1.049}{s^{2}+1.653 s+1.069}, \\
G_{4}(s)=\frac{-1.979 s+0.806}{s^{2}+1.493 s+0.8255}, \\
G_{5}(s)=\frac{-2.002 s+0.72}{s^{2}+1.43 s+0.74} .
\end{gathered}
$$

Step 1 (multimodel control). In first step, multimodel control approach will be used for hydraulic turbine control. For each transfer function, PI controller will be designed using 


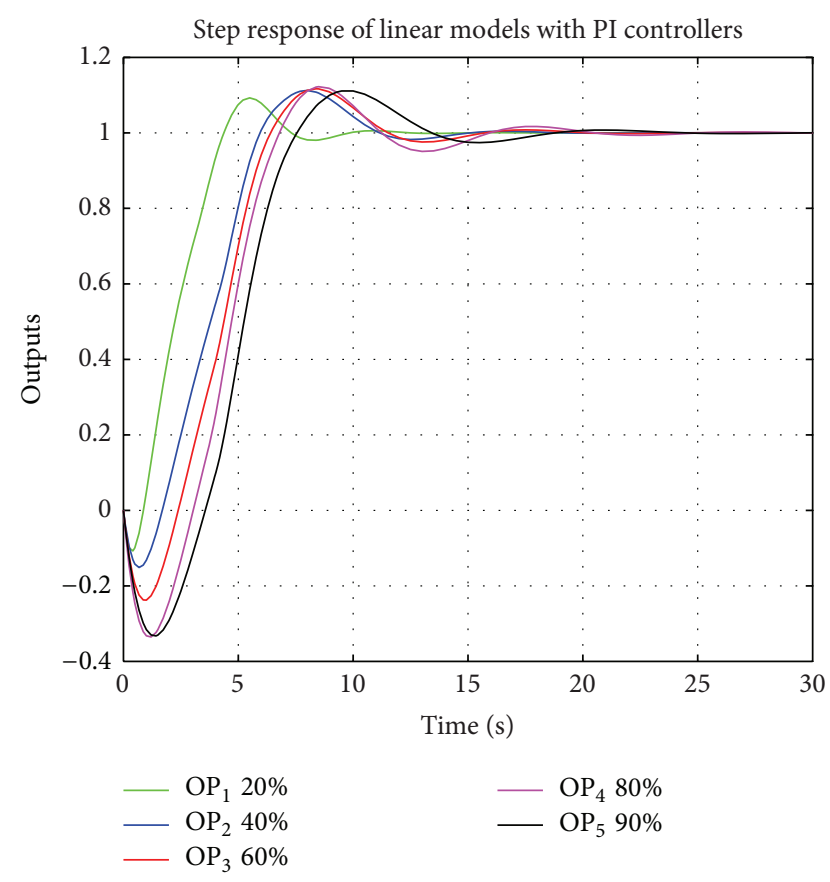

FIGURE 7: Step responses of linear models with PI controllers.

TABLE 1: Switching algorithm based on system output.

\begin{tabular}{lccccc}
\hline Output [\%] & $<0,30)$ & $<30,50)$ & $<50,70)$ & $<70,85)$ & $<85,100)$ \\
\hline Active contr. & $R_{1}(s)$ & $R_{2}(s)$ & $R_{3}(s)$ & $R_{4}(s)$ & $R_{5}(s)$
\end{tabular}

D-partition method [9]. Aim of controller tuning will be keeping overshoot up to $10 \%$ :

$$
\begin{array}{cc}
R_{1}(s)=\frac{0.444 s+0.635}{s}, & R_{2}(s)=\frac{0.263 s+0.36}{s}, \\
R_{3}(s)=\frac{0.306 s+0.275}{s}, & R_{4}(s)=\frac{0.354 s+0.225}{s}, \\
R_{5}(s)=\frac{0.29 s+0.196}{s} .
\end{array}
$$

Figure 7 shows that overshoot condition is satisfied for all linear models (operating points). On the nonlinear system, two different switching algorithms will be tested.

First switching algorithm will be based on system output. Since system output will be lower than $30 \%$, first controller will be active. If output will be more than $30 \%$ but less than $50 \%$, second controller will be active and so on (Table 1 ).

Second algorithm will be based on comparison of system output $y$ and model outputs $y_{q}$ :

$$
J_{q}=y-y_{q}, \quad q=1,2, \ldots, m .
$$

In each sample time comparison will be done and $R_{i}(s)$ controller will be set as active, where $i \in q$ is index of model with smallest $J(20)$.

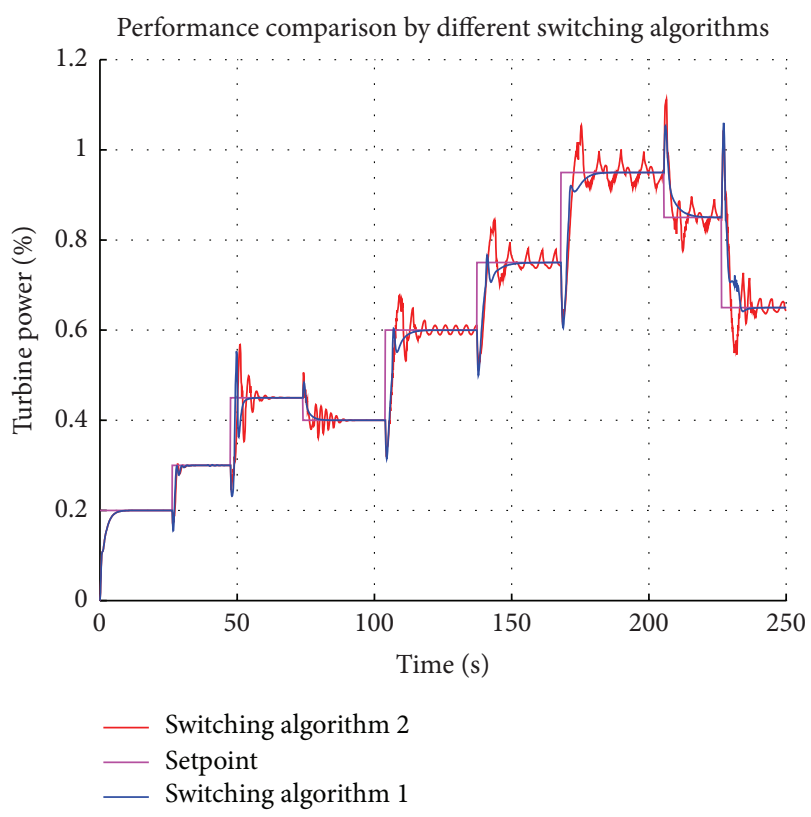

FIGURE 8: Control performance comparison by different switching algorithms.

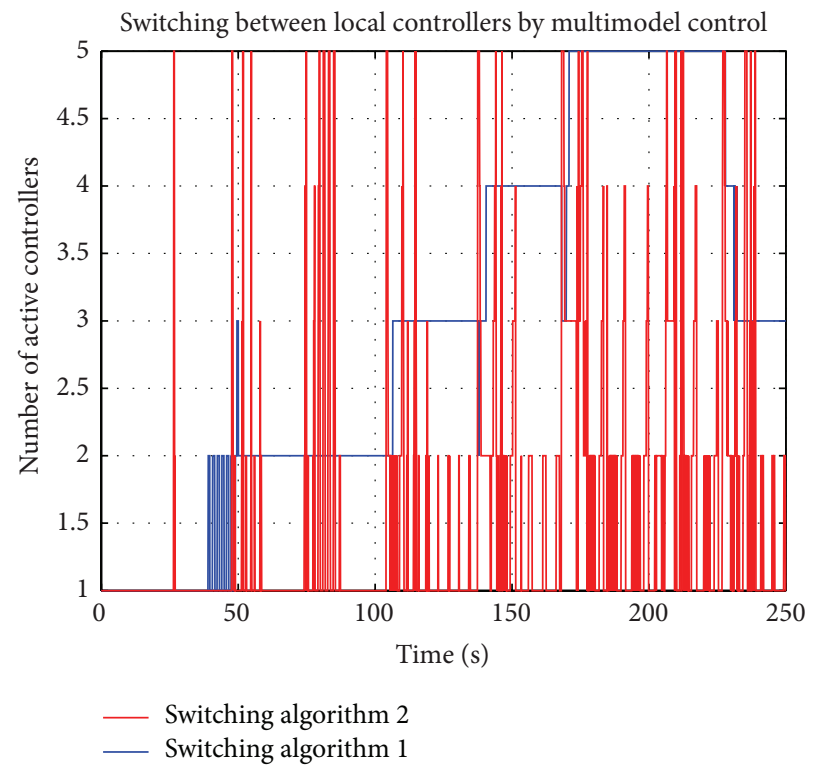

FIGURE 9: Switching between controllers $R_{1}(s)-R_{5}(s)$, comparison of two algorithms.

Nonlinear turbine model has strong nonminimal phase behavior. Performance comparison (Figure 8) by different switching algorithms shows that switching speed has significant influence on performance. First switching algorithm is slow and by second algorithm switching is very fast (Figure 9).

When high control performance is needed, it is recommended to avoid fast switching. Possible solutions could be increasing switching period (not at each sample time), 


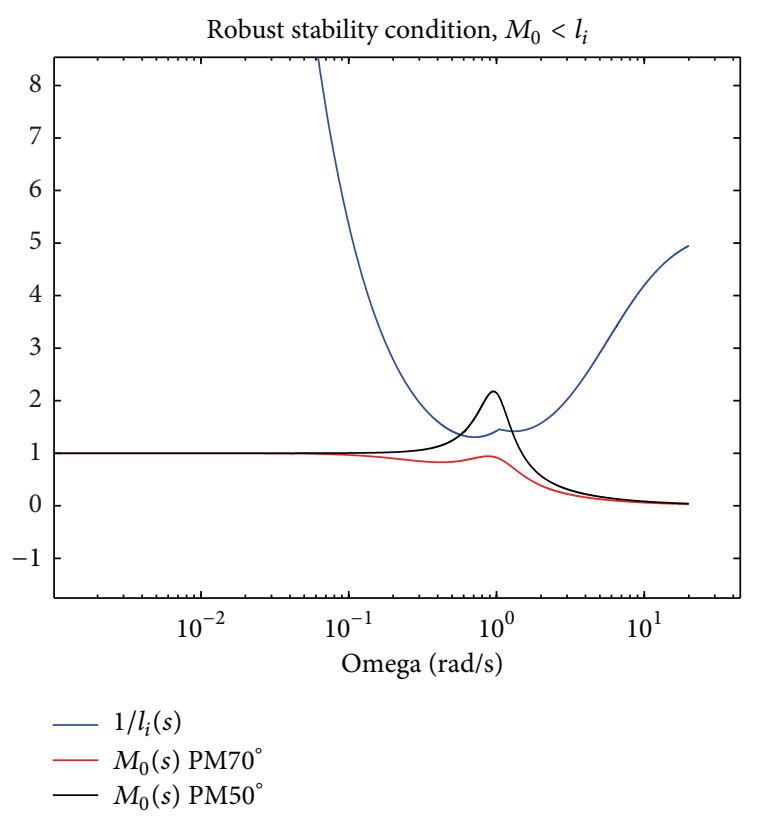

FIGURE 10: Robust stability condition for controllers ensuring phase margins $\mathrm{PM}=50^{\circ}$ and $\mathrm{PM}=70^{\circ}$.

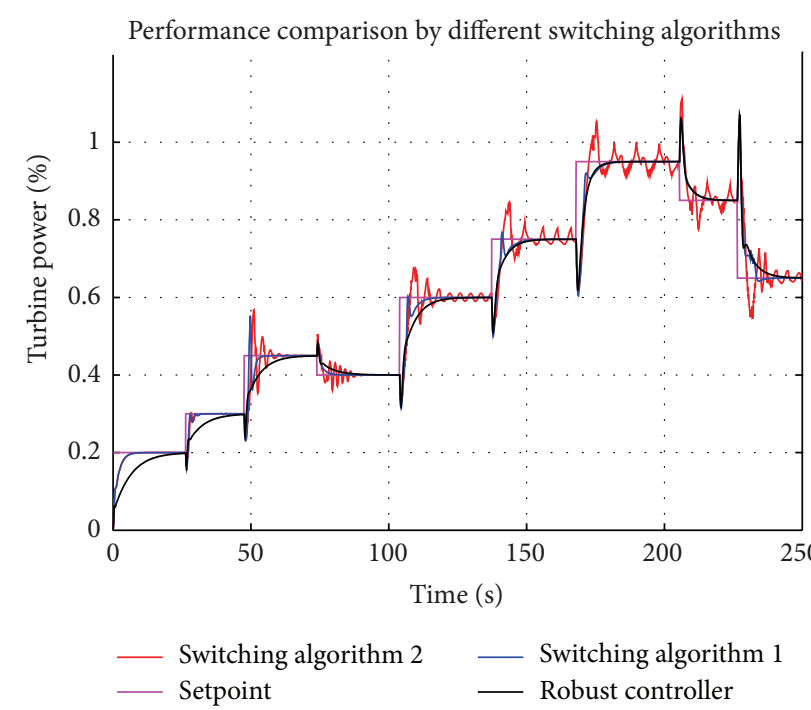

FIGURE 11: Control performance comparison multimodel and robust control.

weighting of controller outputs, or, in this case, using first switching algorithm.

Step 2 (robust control). In second step by robust control design instructions from previous chapter will be used.

From transfer functions (18), nominal model $G_{0}(s)$ will be calculated as transfer function with the same structure and average values:

$$
G_{0}(s)=\frac{-1.868 s+1.3145}{s^{2}+1.833 s+1.3324}
$$

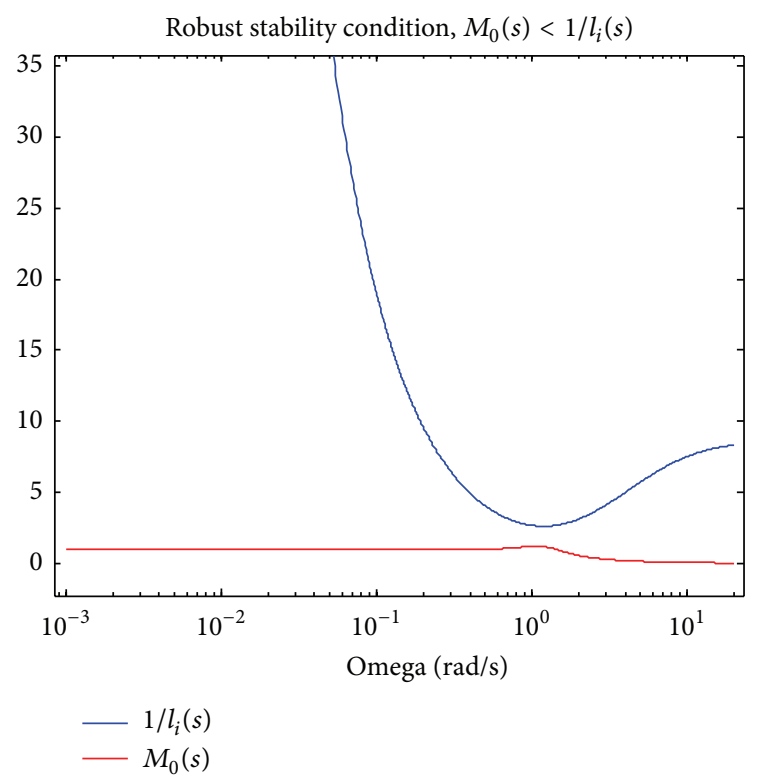

FIGURE 12: Robust stability condition, first operating point.

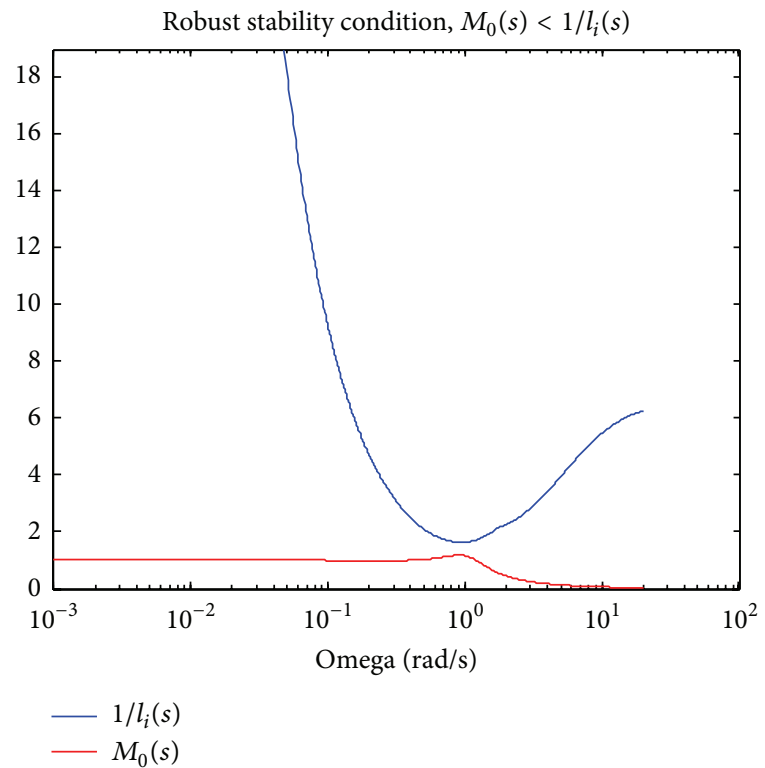

FIGURE 13: Robust stability condition, second operating point.

For nominal model, PI controller was calculated using design method ensuring desired phase margin. Controller ensuring phase margin $\mathrm{PM}=50^{\circ}$ does not guarantee robust stability condition, because desired phase margin was increased to $\mathrm{PM}=70^{\circ}$ (Figure 10).

Transfer function of controller ensuring phase margin $\mathrm{PM}=70^{\circ}$ is

$$
R(s)=\frac{0.3233 s+0.2287}{s} .
$$

Comparison of multimodel and robust control (Figure 11) shows slower response of system with robust control in lower power output. By higher power output, system response with 


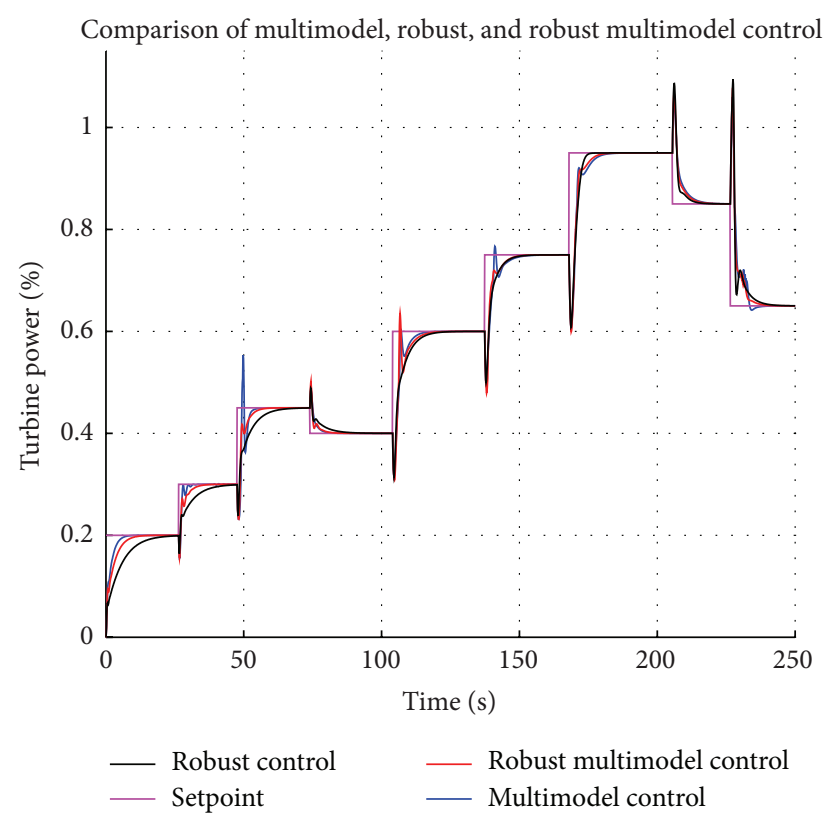

FIGURE 14: Comparison of multimodel, robust, and robust multimodel control.

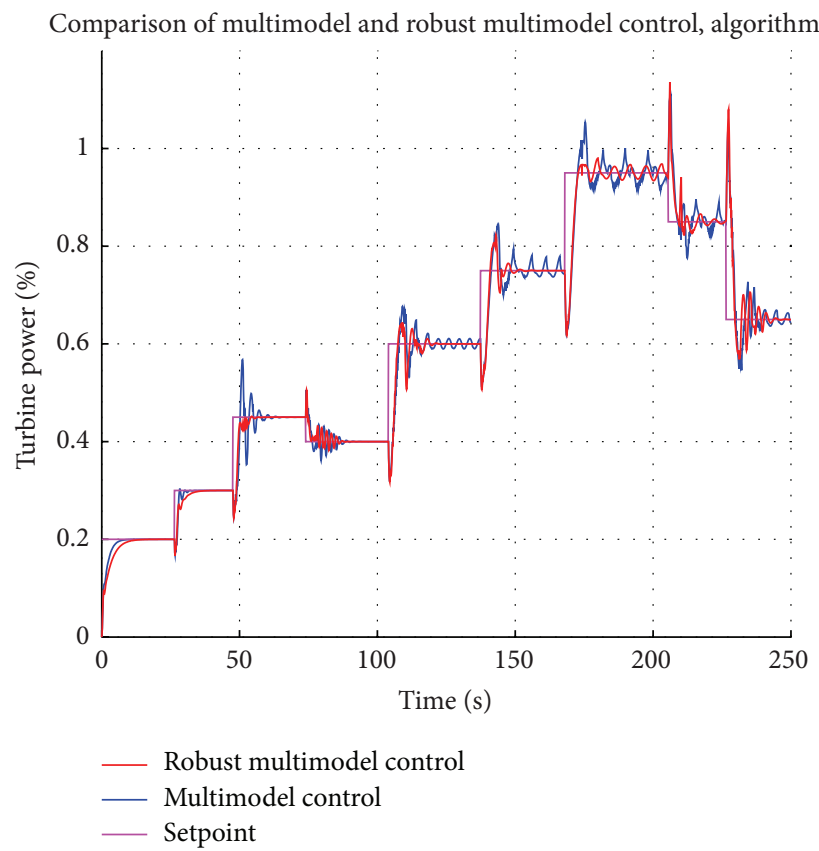

FIGURE 15: Comparison of multimodel and robust multimodel control using second switching algorithm.

TABLE 2: IAE comparison of multimodel control (two switching algorithms) and robust control.

\begin{tabular}{lccc}
\hline Control & Multimodel 1 & Multimodel 2 & Robust \\
\hline IAE & 4,82 & 7,06 & 5,94 \\
\hline
\end{tabular}

robust control is similar to system response with multimodel control. Performance of control approaches will be compared also numerically in Table 2, using IAE criterion.

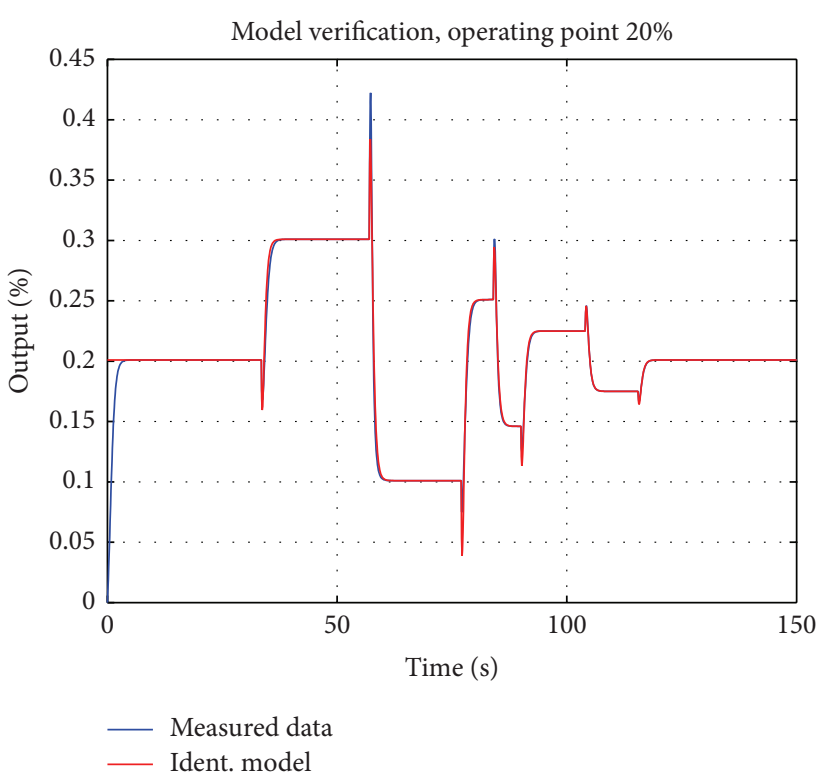

FIGURE 16: Comparison of data measured on nonlinear system and on identified linear model, OP 20\%.

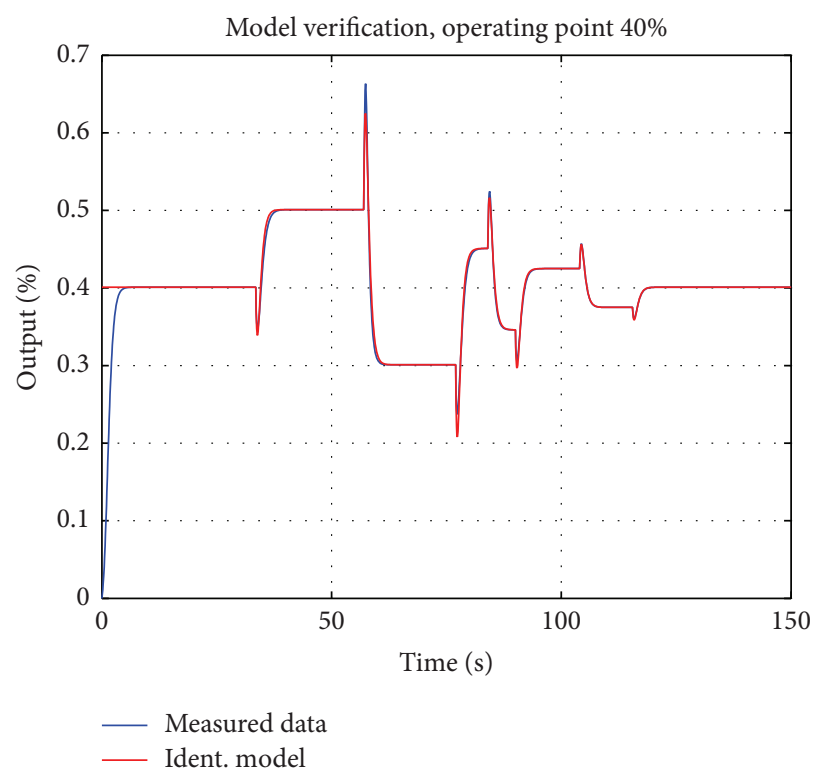

FIgURE 17: Comparison of data measured on nonlinear system and on identified linear model, OP 40\%.

TABLE 3: IAE comparison of multimodel control, robust control, and robust multimodel control.

\begin{tabular}{lccc}
\hline Control & Multimodel 1 & Robust & Multimodel robust \\
\hline IAE & 4,82 & 5,94 & 4,92 \\
\hline
\end{tabular}

Step 3 (robust multimodel control). In third step, robust multimodel control will be designed, which is a combination of robust and multimodel control (see Table 4). 


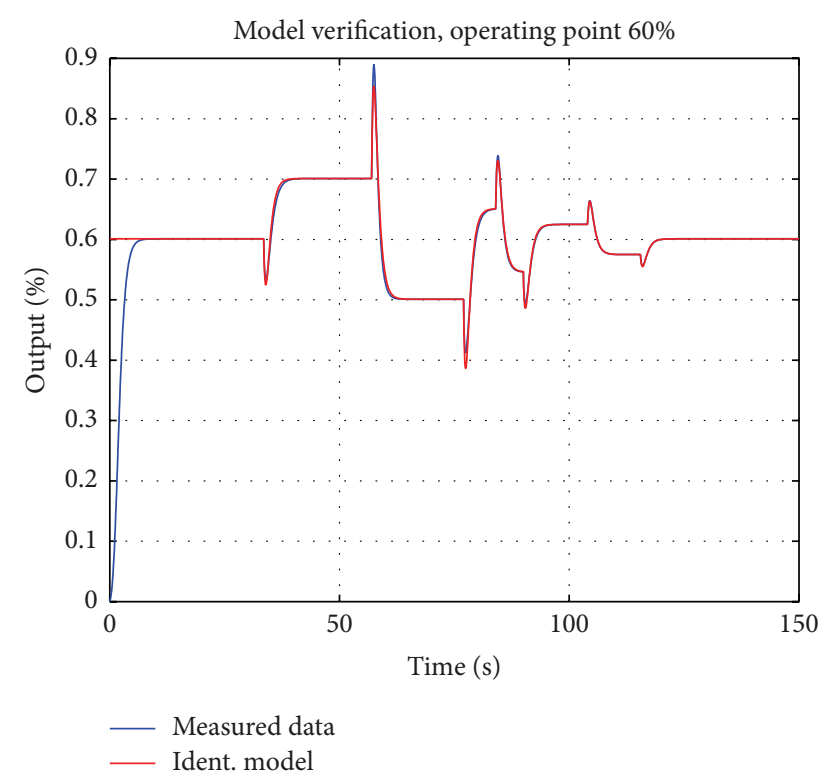

FIGURE 18: Comparison of data measured on nonlinear system and on identified linear model, OP 60\%.

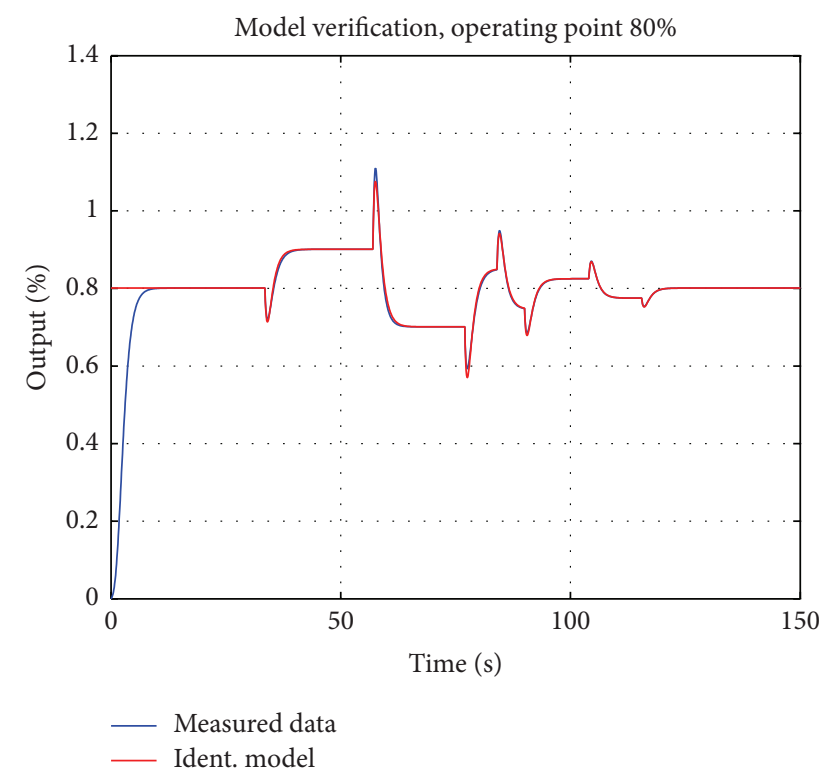

FIGURE 19: Comparison of data measured on nonlinear system and on identified linear model, OP 80\%.

TABLE 4: IAE comparison of multimodel and robust multimodel control algorithm 2.

\begin{tabular}{lcc}
\hline Control algorithm 2 & Multimodel & Robust multimodel \\
\hline IAE & 7,06 & 5,7 \\
\hline
\end{tabular}

By controller design for first operating point, nominal model $G_{0 n}(s)$ is calculated only from $G_{1}(s)$ and $G_{2}(s)$ according to (18):

$$
G_{0 n 1}(s)=\frac{-1.714 s+1.99}{s^{2}+2.29 s+2.014}
$$

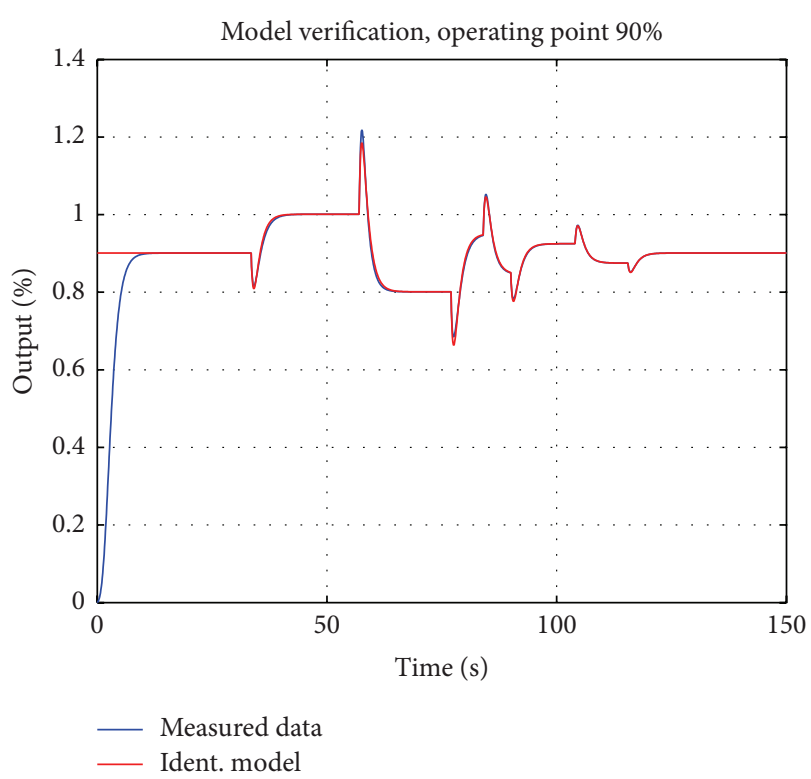

FIGURE 20: Comparison of data measured on nonlinear system and on identified linear model, OP 90\%.

For this nominal model controller ensuring phase margin $\mathrm{PM}=60^{\circ}$ will be designed:

$$
R_{1}(s)=\frac{0.4275 s+0.434}{s}
$$

Robust stability condition (Figure 12) proves robust properties of designed controller. From behaviour of $M_{0}(s)$ (in Figures 10 and 12), it is obvious that that robust multimodel control is less conservative as pure robust control.

By controller design for second operating point, nominal model $G_{0 n}(s)$ is calculated from $G_{1}(s), G_{2}(s)$, and $G_{3}(s)$ according to (18):

$$
G_{0 n 2}(s)=\frac{-1.786 s+1.682}{s^{2}+2.08 s+1.7}
$$

For this nominal model controller ensuring phase margin $\mathrm{PM}=60^{\circ}$ will be designed:

$$
R_{2}(s)=\frac{0.375 s+0.429}{s} .
$$

Robust stability condition (Figure 13) proves robust properties of designed controller.

Controller design for the rest of operating points goes the same way. Controller parameters for operating points 3-5 are as follows:

$$
\begin{gathered}
R_{3}(s)=\frac{0.34 s+0.251}{s}, \quad R_{4}(s)=\frac{0.297 s+0.23}{s}, \\
R_{5}(s)=\frac{0.305 s+0.208}{s} .
\end{gathered}
$$

Comparison of three control approaches (Figure 14) shows that robust multimodel control is almost so fast as multimodel control. Performance of multimodel, robust, and 
robust multimodel control is compared in Table 3 by first switching algorithm (described in Table 1).

Performance of multimodel and robust multimodel approaches will be compared also using second switching algorithm (Figure 15). Robust properties improved performance by this fast switching algorithm.

\section{Conclusion}

In this paper, hybrid control, often denoted by multimodel control, was used. Two switching algorithms were compared to show how the switching speed affects performance and stability. Robust control method based on $M-\Delta$ was presented as a control solution which ensures stability on whole operating range, but with lower performance. Combination of multimodel control and robust control which ensure stability and good performance was illustrated on nonlinear hydraulic turbine model. Designed control solution is less conservative as robust control but stable on whole operating range and almost with the same performance as using multimodel control.

\section{Appendix}

For identification results, in form of model verification, in all operating points, see Figures 16-20.

\section{Conflict of Interests}

The authors declare that there is no conflict of interests regarding the publication of this paper.

\section{Acknowledgment}

This work was supported by the Scientific Grant APVV 077212.

\section{References}

[1] T. Puleva, E. Garipov, and G. Ruzhekov, "Adaptive power control modeling and simulation of a hydraulic turbine," in Proceedings of the16th International Conference on Intelligent System Applications to Power Systems (ISAP '11), September 2011.

[2] O. P. Malik and Y. Zeng, "Design of a robust adaptive controller for a water turbine governing system," IEEE Transactions on Energy Conversion, vol. 10, no. 2, pp. 354-359, 1995.

[3] J. Lunze and F. L. Lagarrigue, Hanbook of Hybrid Systems Control, Theory, Tools, Applications, Cambridge University Press, New York, NY, USA, 2009.

[4] M. S. Branicky, V. S. Borkar, and S. K. Mitter, "A unified framework for hybrid control: model and optimal control theory," IEEE Transactions on Automatic Control, vol. 43, no. 1, pp. 31-45, 1998.

[5] J. Lygeros, "An overview of research areas in hybrid control," in Proceedings of the 44th IEEE Conference on Decision and Control, and European Control Conference (CDC-ECC '05), pp. 5600-5605, December 2005.
[6] R. Goebel and A. R. Teel, "Solutions to hybrid inclusions via set and graphical convergence with stability theory applications," Automatica, vol. 42, no. 4, pp. 573-587, 2006.

[7] D. Liberzon, Switching in Systems and Control, Birkhauser, Boston, Mass, USA, 2003.

[8] S. Skogestad and I. Postlethwaite, Multivariable Feedback Control: Analysis and Design, John Wiley \& Sons, New York, NY, USA, 3rd edition, 1997.

[9] J. Osuský and V. Veselý, "Modification of Neimark D-partition method for desired phase margin," Journal of Cybernetics and Informatics, vol. 11, 2010. 

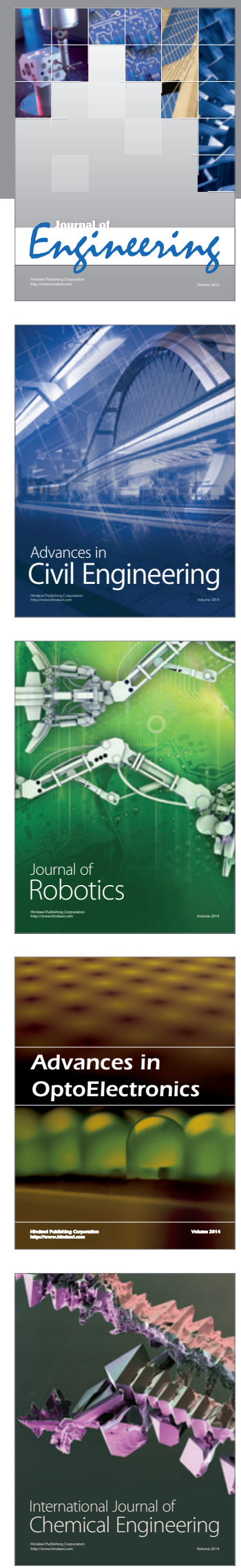

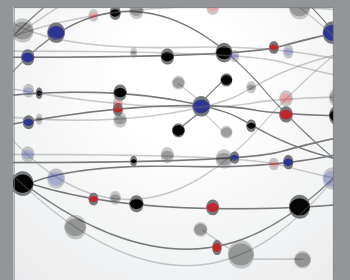

The Scientific World Journal
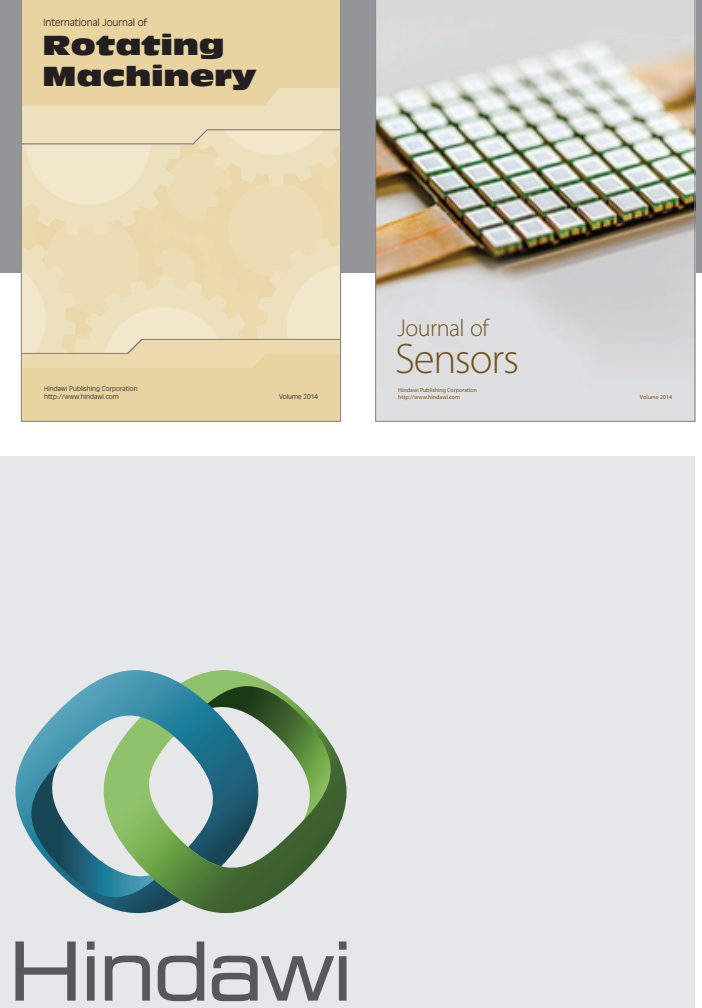

Submit your manuscripts at http://www.hindawi.com
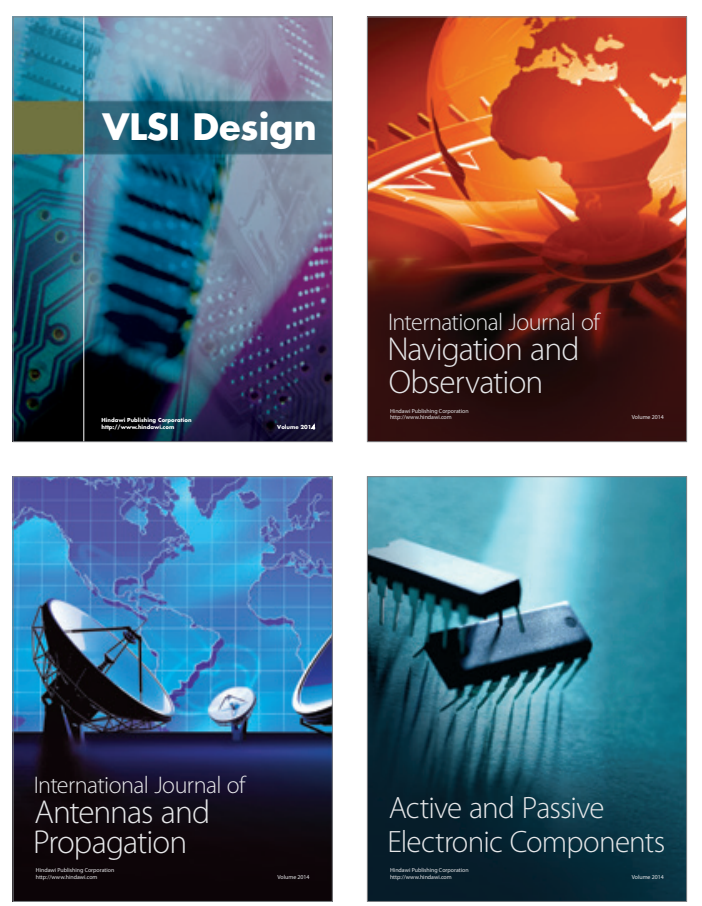
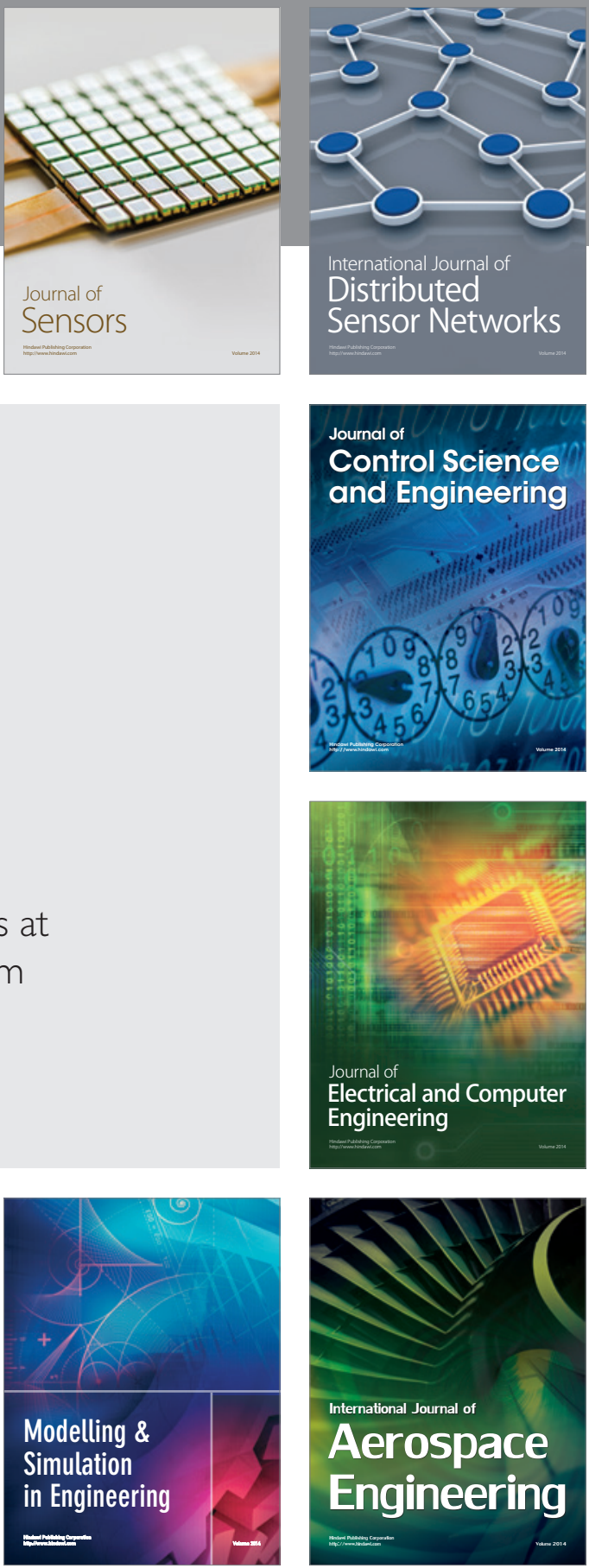

Journal of

Control Science

and Engineering
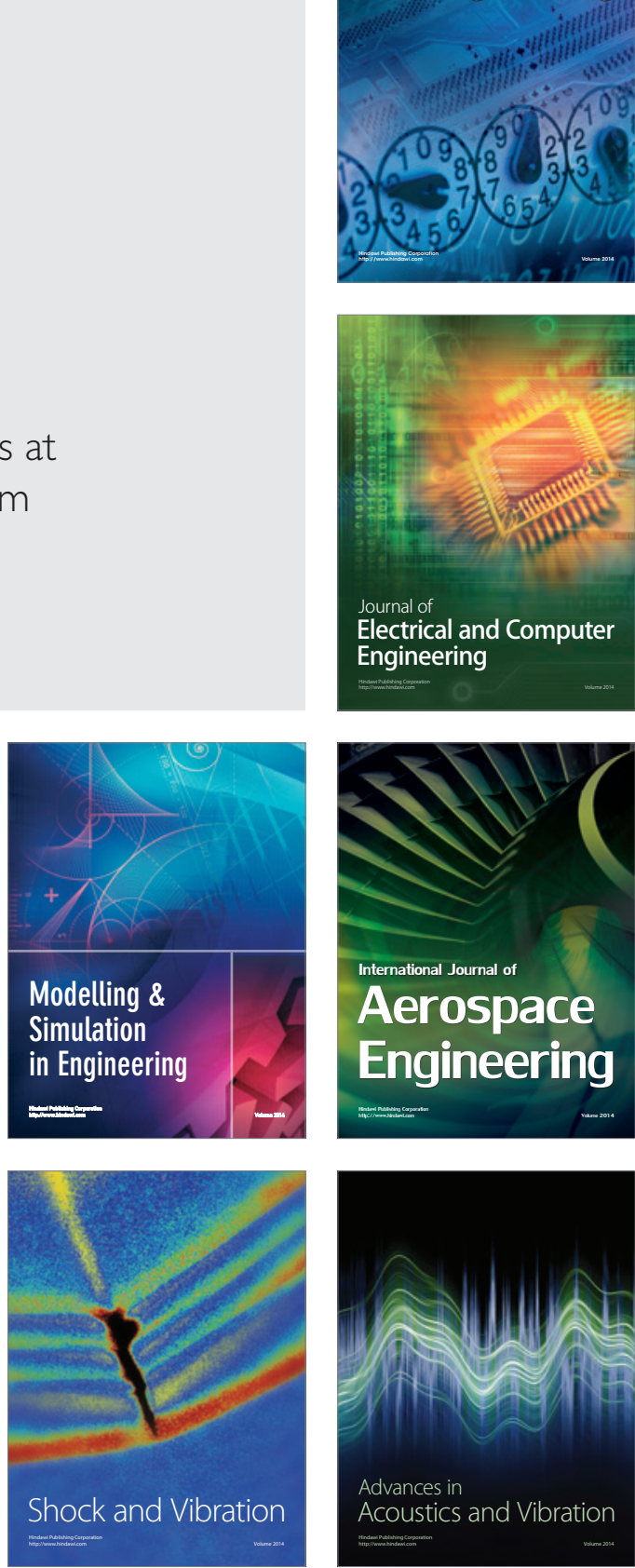